(2) Open Access Full Text Article

\title{
Agreement and relationship between ultrasonic and partial coherence interferometry measurements of axial length and anterior chamber depth
}

This article was published in the following Dove Press journal:

Clinical Ophthalmology

27 January 2012

Number of times this article has been viewed

\section{Amany R Wissa \\ Sherein S Wahba \\ Maged M Roshdy}

Ain Shams University and National Eye Hospital, Cairo, Egypt
Correspondence: Sherein Shafik Wahba 6 Mohamed Shoukry Street, Gesr El Suez, Al Zaitoun, Cairo, Egypt

Tel +20 I22242 I896

Email s_shafik@hotmail.com
Purpose: To find the relationship between axial length (AL) and anterior chamber depth (ACD) measurements, using partial coherence interferometry (PCI) and A-scan ultrasonography (US).

Setting: National Eye Hospital, Cairo, Egypt.

Method: Retrieving and comparing biometric data from the files of 163 consecutive patients seeking cataract extraction by PCI (IOLMaster) and US (Sonomed).

Results: AL measured using US range from 20.93 to $33.17 \mathrm{~mm}$ (mean $\pm \mathrm{SD}=24.45 \pm 2.73 \mathrm{~mm}$ ). AL measured by PCI range from 20.90 to $33.27 \mathrm{~mm}(24.05 \pm 2.76 \mathrm{~mm})$. The range of ACD measured by US was 2.09 to $4.48 \mathrm{~mm}$ (3.32 $\pm 0.46 \mathrm{~mm})$. The range of ACD measured by PCI was 2.15 to $4.29 \mathrm{~mm}(3.31 \pm 0.45 \mathrm{~mm})$. There is very high agreement between both methods; the intraclass correlation coefficient $=0.999$ for $\mathrm{AL}$, and 0.966 for ACD measurements. A linear regression model of two formulae fits the AL values (one for eyes longer than $29 \mathrm{~mm}$, and the other for the shorter eyes), with no significant departure from linearity $(P>0.1)$. One formula fits the ACD values with significant departure from linearity $(P<0.05)$.

Conclusion: Both US and PCI methods for measurements of AL and ACD are highly correlated. Therefore, the value of AL measured by one method can be predicted, with high accuracy, from the other method.

Keywords: axial length, anterior chamber depth, A-scan US, partial coherence interferometry

\section{Introduction}

New intraocular lenses (IOLs) such as accommodative, multifocal, and aspheric models need accurate ocular biometry for accurate IOL power calculations. Otherwise, the patients may be left with a significant refractive error. ${ }^{1}$ Accurate axial length (AL) measurement has been shown to be the most important parameter for IOL power determination. $^{2}$ On the other hand, anterior chamber depth (ACD) measurement is required by several newer theoretical IOL power formulas to fine-tune the effective lens position; therefore, its accurate measurement is essential to minimize the risk of unwanted refractive outcomes. ${ }^{1}$ Several noncontact biometry devices compare favorably to older ultrasonic biometric and keratometric techniques. ${ }^{3} \mathrm{~A}$-scan US measurement of the AL has a longitudinal resolution of $200 \mu \mathrm{m}$ and an accuracy of 100-200 $\mu \mathrm{m} .{ }^{4} \mathrm{An}$ error of $100 \mu \mathrm{m}$ in AL measurements leads to about 0.28 diopter (D) of postoperative refractive error. ${ }^{5}$

In 1999, Carl Zeiss Meditec (Jena, Germany) introduced a noncontact partial coherence laser interferometer PCI (IOLMaster) as an alternative technique to measure the AL. ${ }^{1}$ The PCI provides three different measurements: the AL, the phakic ACD, 
and the keratometric $(\mathrm{K})$ readings. This set of measurements is sufficient to predict IOL power. The measurement of the AL is based on PCI using a laser diode in the near infrared spectrum (780 nm). This technique is able to measure AL with a high resolution of $12 \mu \mathrm{m}$ and very high precision of $0.3-10 \mu \mathrm{m}$ along the fixation line of the eye. ${ }^{6-8}$ Advantages over conventional ultrasound (US) include: high precision (reproducibility), contact-free measurement, and observer independence of the measurements. ${ }^{9,10}$

Moreover, the AL measurement is performed through the visual axis since the patient is asked to fixate on the laser spot. In highly myopic or staphylomatous eyes, this can be of particular advantage since it can sometimes be difficult to measure the true AL through the visual axis with an ultrasonic probe. Optical biometry is also superior to US in the measurement of the pseudophakic and silicone oil-filled eye because the correction factor needed is much smaller than in ultrasonic biometry. ${ }^{11}$

The aim of this study was to investigate the agreement between contact A-scan US and PCI measurements of AL and ACD. In addition, we tried to find a formula with high accuracy to convert data between the two methods.

\section{Materials and methods}

This retrospective study included 163 eyes for AL measurements and 104 eyes for ACD measurements of patients asking for cataract surgery at the National Eye Hospital (Cairo, Egypt). Data were collected retrospectively from patients' files including age, gender, history, clinical findings, PCI examination, and contact A-scan US results. All candidates were examined by the same ophthalmologist.

Exclusion criteria included patients with corneal opacification, previous corneal surgery, corneal edema, previous documented trauma cases, anterior segment inflammation, clinically diagnosed keratoconus and a history of glaucoma. In addition, patients were excluded if they had factors that could cause questionable measurement by PCI as a result of retinal detachment, silicone oil-filled eye, dense cataract, etcetera. Preoperatively, all candidates underwent PCI by IOLMaster (with software v4.08) and a contact A-scan ultrasound by Sonomed A/B Scan 5500 (Sonomed, Lake Success, NY) using the contact (applanation) technique to determine $\mathrm{AL}, \mathrm{K}$ readings and $\mathrm{ACD}$.

\section{Statistical analysis}

Data were collected, revised, and analyzed using Microsoft Office Excel (2007; Redmond, WA), IBM SPSS Statistics (v19; Armonk, NY), and MedCalc (v11.1.1.0; Medcalc Software bvba, Ghent, Belgium). The following were calculated: the mean, standard deviation (SD), standard error of the mean (SEM), intraclass correlation coefficient, cusum test, $t$-test, Welch test, ANOVA test, post hoc test, and the linear regression. A $P$-value of less than 0.05 indicated statistical significance.

A scatter graph and Pearson's correlation coefficient were used to investigate the linear relationship between the AL and ACD by PCI versus those by contact A-scan ultrasound.

The agreement between the two methods was investigated using a Bland-Altman plot, which is a graph of the ratios between readings measured by the two methods plotted against the means for the pairs of measurements. The upper and lower limits for the $95 \%$ confidence interval for the mean differences are connected with horizontal straight lines, thus providing a band that helps to visualize the extent of agreement between the two methods.

\section{Results}

\section{Demographic data: (Table I)}

163 measurements of AL, and 104 measurements of ACD:

- The mean age of the patients was $64.3 \pm 10.9$ years (ranging from 35-87.5 years).

- The mean IOL power was $18.1 \pm 7.5$ diopters (ranging from -5 to 30 diopters).

- The right eye to left eye ratio was 80:83.

- The male to female ratio was 64:99.

\section{AL measurements: (Table 2)}

(a) By contact A-scan US:

- Range: 20.93-33.17 mm

- Mean \pm SD: $24.45 \pm 2.73 \mathrm{~mm}$

(b) By PCI:

- Range: 20.90-33.27 mm

- Mean \pm SD: $24.05 \pm 2.76 \mathrm{~mm}$.

There was a highly significant intraclass correlation coefficient between the PCI and contact A-scan US measurements for AL with the average for absolute agreement $=0.999$ $($ Pearson Correlation $=0.998)($ Figure 1$)$.

Bland-Altman plots were created to assess the difference in individual measurement as a function of the mean

Table I Demographic data

\begin{tabular}{lll}
\hline & Mean \pm SD & Range \\
\hline Age & $64.3 \pm 10.9$ y & $35-87.5 \mathrm{y}$ \\
IOLs power & $18.1 \pm 7.5 \mathrm{D}$ & $-5-+30 \mathrm{D}$ \\
Numbers & & \\
Side & 80 right & $83 \mathrm{left}$ \\
Gender & 64 male & $99 \mathrm{female}$ \\
\hline
\end{tabular}

Abbreviations: IOLs, intraocular lenses; SD, standard deviation; $y$, years. 
Table 2 Mean AL and ACD measurements by both US and $\mathrm{PCl}$

\begin{tabular}{lll}
\hline & US (in mm) & PCl (in mm) \\
\hline AL & & \\
$\quad$ Range & $20.93-33.17$ & $20.90-33.27$ \\
$\quad$ Mean \pm SD & $24.45 \pm 2.73$ & $24.05 \pm 2.76$ \\
ACD & & \\
Range & $2.09-4.48$ & $2.15-4.29$ \\
Mean \pm SD & $3.32 \pm 0.46$ & $3.31 \pm 0.45$ \\
\hline
\end{tabular}

Abbreviations: AL, axial length; $A C D$, anterior chamber depth; US, ultrasound $\mathrm{PCl}$, partial coherence interferometry; SD, standard deviation.

of the two measurements for that subject. The two methods showed good agreement, $95 \%$ of the differences in the readings between them lay between $0.992 \mathrm{~mm}$ and $1.017 \mathrm{~mm}$ (Figure 2).

The difference between AL measurements with PCI and that with US:

- There was a mean difference in the measured AL obtained with PCI and contact A-scan US of $0.11 \mathrm{~mm}$ (range -0.47 to $0.72 \mathrm{~mm}$ ).

- It doesn't depend on gender $(t=-0.033, P=0.903)$ by Welch test.

- It doesn’t depend on laterality $(t=1.374, P=0.172)$ by Student $t$-test.

- It doesn't correlate with age $(r=-0.010, P=0.090)$ by Pearson's correlation coefficient.

- It correlates with AL itself ( $r=0.0230, P=0.003)$ by Pearson's correlation coefficient.

- The ANOVA analysis showed significant differences $(F$-ratio $=9.965, P<0.001)$. The Student-NewmanKeuls test for all corresponding comparisons showed that the long AL group is different from both the short and medium AL groups, which showed no significant difference between them (Table 3).

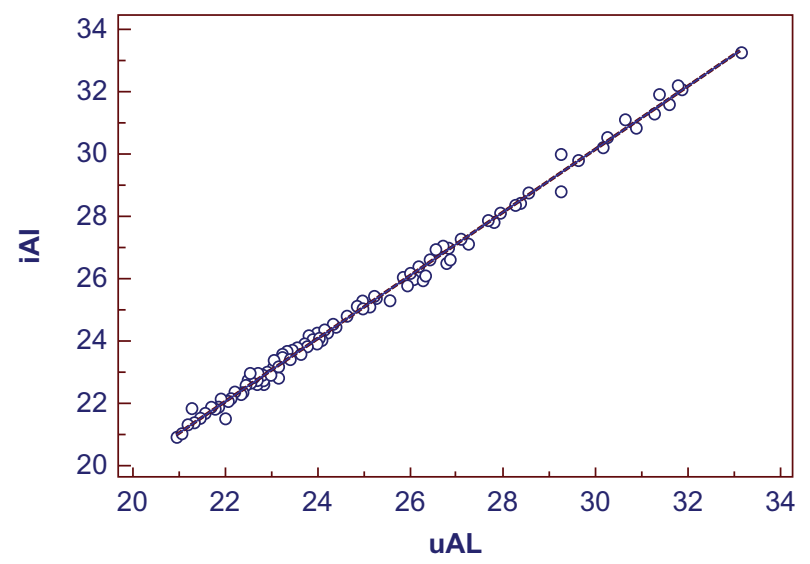

Figure I Intraclass correlation coefficient between the $\mathrm{PCl}$ and contact A-scan ultrasonograph measurements for $\mathrm{AL}$

Abbreviations: $\mathrm{iAl}, \mathrm{AL}$ by partial coherence interferometry; $\mathrm{uAL}, \mathrm{AL}$ by ultrasonography; $\mathrm{PCl}$, partial coherence interferometry; $\mathrm{AL}$, axial length.
Therefore, by linear regression we can only adopt two formulae:

1. For eyes with AL less than or equal to $29 \mathrm{~mm}$ by PCI:

Equation: $\mathrm{AL}(\mathrm{PCI})=0.2824+0.9919 \mathrm{AL}$ (US)

$\mathrm{R}^{2}=0.9938, P<0.001$

2. For eyes with AL more than $29 \mathrm{~mm}$ by PCI:

Equation: $\mathrm{AL}(\mathrm{PCI})=2.6108+0.9238 \mathrm{AL}(\mathrm{US})$

$\mathrm{R}^{2}=0.9564, P<0.001$

Cusum test for linearity: there is no deviation from linearity $(P>0.1)$.

\section{ACD measurements: (Table 2)}

(a) By contact A-scan US:

- Range: 2.09-4.48 mm

- Mean \pm SD: $3.32 \pm 0.46 \mathrm{~mm}$

(b) By PCI:

- Range: $2.15-4.29 \mathrm{~mm}$

- Mean \pm SD: $3.31 \pm 0.45 \mathrm{~mm}$

There was a highly significant intraclass correlation coefficient between the PCI and contact A-scan US measurements for ACD, with the average for absolute agreement $=0.968$ (Pearson's correlation coefficient $=0.938)$ (Figure 3).

From the Bland-Altman analysis, the two methods showed good agreement. Ninety-five percent of the differences in the readings between them lay between 0.91 and 1.10 (Figure 4).

The difference between ACD measurements with PCI and measurements with US:

- There was a mean difference in the measured ACD obtained with PCI and contact A-scan US of $0.01 \mathrm{~mm}$ (range $-0.53-0.56 \mathrm{~mm}$ ).

- It doesn't depend on gender $(t=0.000, P=1.000)$ by Student $t$-test.

- It doesn't depend on laterality ( $t=0.495, P=0.622)$ by Student $t$-test.

- It doesn't correlate with age $(r=-0.079, P=0.424)$ by Pearson's correlation coefficient.

- It correlates poorly with AL $(r=0.185, P=0.060)$ by Pearson's correlation coefficient (Table 4).

- The ANOVA analysis showed no significant differences $(F$-ratio $=1.250, P=0.291)$.

Therefore, by linear regression we can adopt one formula only:

- Equation: ACD (PCI) $=0.2788+0.9208$ ACD (US) $\mathrm{R}^{2}=0.8734, P<0.001$

- Cusum test for linearity: there is a significant deviation from linearity $(P<0.05)$. 


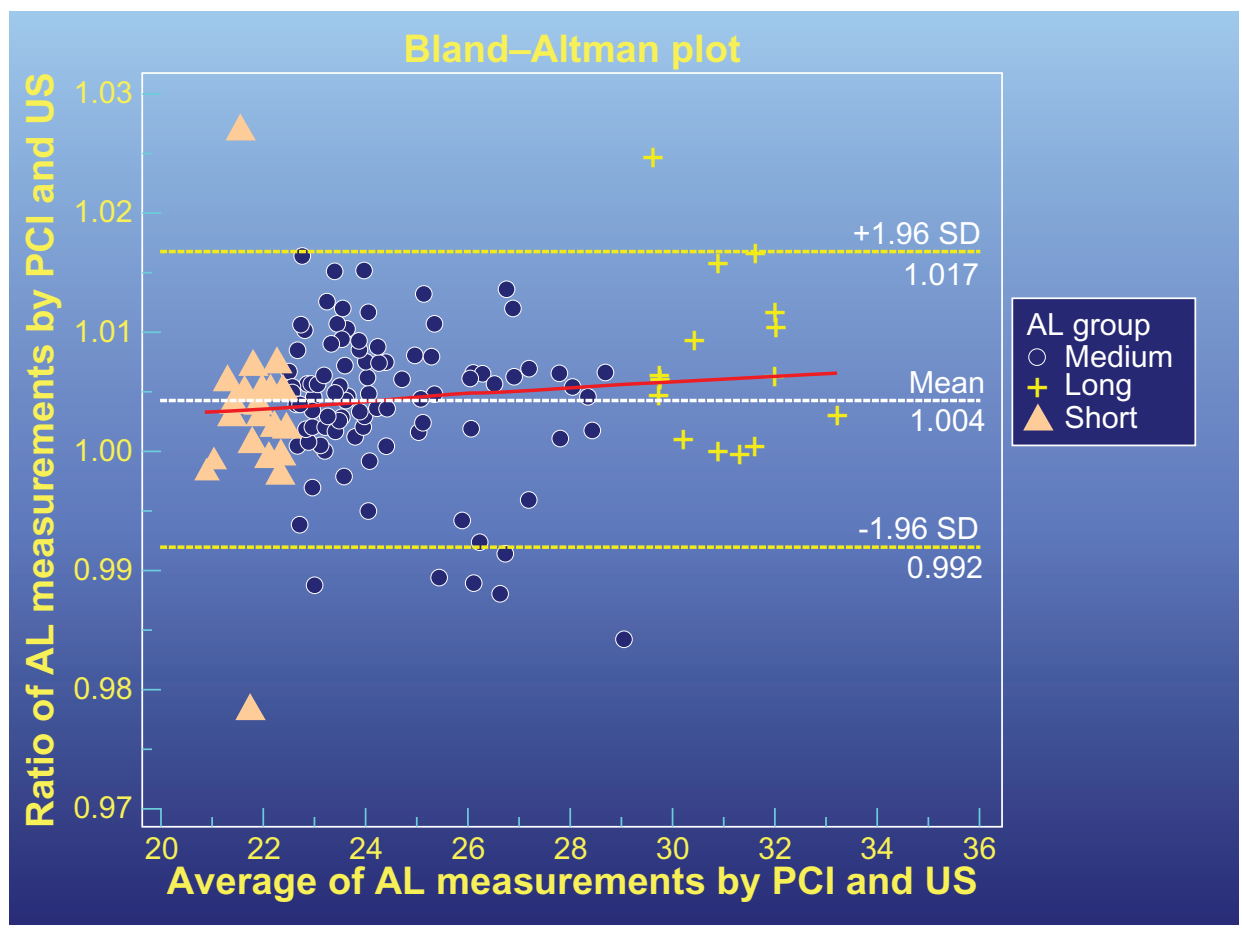

Figure 2 Bland-Altman plot between the $\mathrm{PCl}$ and contact A-scan ultrasonograph measurements for AL.

Abbreviations: AL, axial length; $\mathrm{PCl}$, partial coherence interferometry; US, ultrasound; SD, standard deviation.

\section{Discussion}

This study showed good agreement, with a mean difference in the measured AL obtained with optical biometry and contact biometry of $0.10 \mathrm{~mm}$ (range $-0.47-0.72 \mathrm{~mm}$ ). Hitzenberger et a ${ }^{12}$ found that the ALs measured by optical biometry were $0.18 \mathrm{~mm}$ longer than those measured by the immersion technique, and $0.47 \mathrm{~mm}$ longer than those measured by the contact technique. Kiss et a ${ }^{13}$ reported a mean difference in the measured AL obtained with optical biometry and immersion biometry of $0.22 \mathrm{~mm}$ (range $-0.24-0.57 \mathrm{~mm}$ ).

Németh et al found that, for 208 eyes, the AL values measured by A-scan ultrasound and by PCI were significantly correlated $(r=0.985, P=0.001)$; however, the PCI values were significantly higher than those of the A-scan US (mean difference $=0.39 \pm 0.36 \mathrm{~mm}) .{ }^{8}$

In the previous studies including the present study, the ALs measured by the optical method were significantly longer

Table 3 The difference between AL measurements with PCL and that with US

\begin{tabular}{lllll}
\hline & Total & Short AL & Medium AL & Long AL \\
\hline Sample size & 163 & 32 & 115 & 16 \\
Range (in mm) & $-0.47-0.72$ & $-0.47-0.58$ & $-0.46-0.37$ & $-0.01-0.72$ \\
Mean \pm SD & $0.11 \pm 0.16$ & $0.06 \pm 0.14$ & $0.10 \pm 0.14$ & $0.27 \pm 0.24$ \\
(in mm) & & & & \\
\hline
\end{tabular}

Abbreviations: AL, axial length; SD, standard deviation. than those measured by US; however, the values obtained by the two methods were closely correlated. The IOLMaster software is calibrated so that the optically measured value is adjusted using a regression model to the value measurable by the immersion US method. ${ }^{14}$

The longer eyes tend to be more compressible in contact A-scan US. This suggests a linear relationship between the magnitude of compression and the AL. Our study found the linear formulas describing the relationship between both methods of AL measurement with a good agreement. Therefore, the IOLMaster software could be

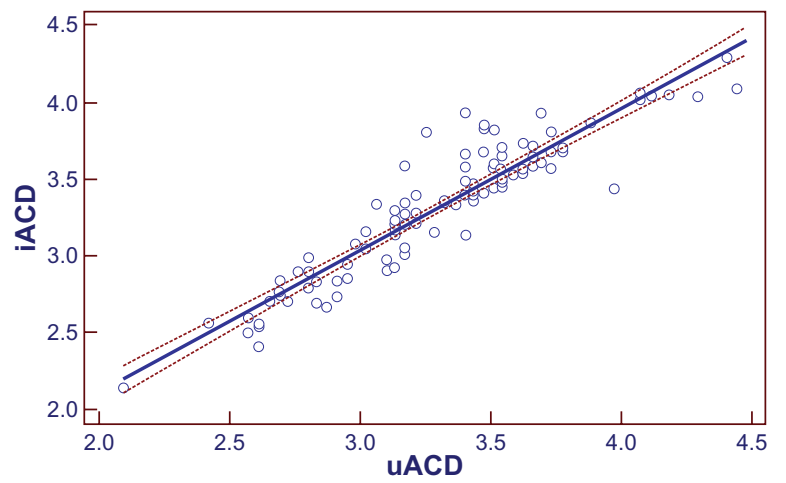

Figure 3 Intraclass correlation coefficient between the $\mathrm{PCl}$ and contact A-scan ultrasonograph measurements for $A C D$.

Abbreviations: $A C D$, anterior chamber depth; $i A C D, A C D$ by partial coherence interferometry; uACD, $\mathrm{ACD}$ by ultrasonography; $\mathrm{PCl}$, partial coherence interferometry. 


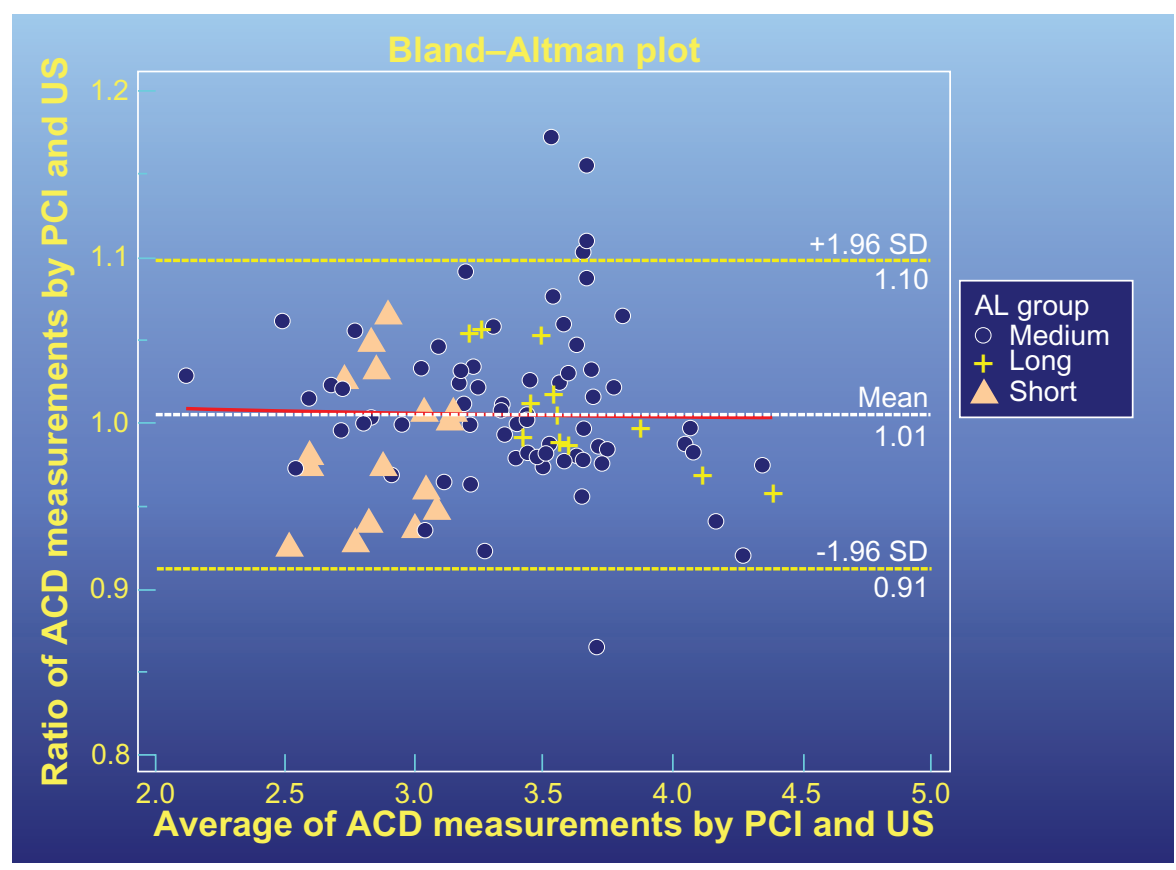

Figure 4 Bland-Altman plot between the $\mathrm{PCl}$ and contact A-scan ultrasonograph measurements for ACD.

Abbreviations: $A C D$, anterior chamber depth; $\mathrm{PCl}$, partial coherence interferometry; US, ultrasound; $\mathrm{AL}$, axial length; SD, standard deviation.

modified to accept A-scan measured AL to calculate the IOL power. The present study reported a good agreement with a mean difference in the measured ACD obtained with optical biometry and contact biometry of $0.014 \mathrm{~mm}$ but with significant deviation from linearity. Németh et al reported that the ACD values with the IOLMaster in 252 eyes were significantly higher (by $0.28 \mathrm{~mm}$ ) than the US values with no correlation between the two sets of values. ${ }^{8}$

Reddy et $\mathrm{al}^{15}$ found that contact US measured ACD is $13 \%$ shorter, while the Orbscan and IOLMaster showed good correlation.

Decentration and misalignment with the visual axis in case of contact A-scan US made the difference between both measurements dependent upon the pupil diameter and accommodative state of the lens. These cause pronounced differences in ACD measurement and could explain the deviation from linearity in the ACD formula.

Table 4 The difference between $A C D$ measurements with $P C I$ and $A C D$ measurements with US

\begin{tabular}{lllll}
\hline & Total & Short AL & Medium AL & Long AL \\
\hline Sample size & 104 & 18 & 74 & 12 \\
Range (in mm) & $-0.53-0.56$ & $-0.2-0.19$ & $-0.53-0.56$ & $-0.19-0.18$ \\
Mean \pm SD & $0.01 \pm 0.16$ & $-0.04 \pm 0.13$ & $0.027 \pm 0.17$ & $0.02 \pm 0.12$ \\
(in mm) & & & & \\
\hline
\end{tabular}

Abbreviations: $A C D$, anterior chamber depth; $\mathrm{PCl}$, partial coherence interferometry; US, ultrasound; $A L$, axial length; SD, standard deviation.
In conclusion, although PCI is generally more accurate, we still need US measurements in some situations (eg, tear film abnormalities, corneal pathology, mature and dense posterior subcapsular cataracts, vitreous opacities, maculopathy, or retinal detachment). ${ }^{1,16}$ It is better to combine US measurements with PCI capabilities. In order to do this, we need AL and ACD measurements to be convertible from one method to the other. This is fulfilled with the linear regression conversion formulae obtained with $99 \%$ accuracy for the AL, and $87 \%$ accuracy in ACD.

\section{Disclosure}

The authors did not receive any financial support from any public or private sources. The authors have no financial or proprietary interests in a product, method, or material described herein.

\section{References}

1. Alexander CL, Mujtaba AQ, Jay SP. Biometry and intraocular lens power calculation. Curr Opin Ophthalmol. 2008;19:13-17.

2. Olsen T. Sources of error in intraocular lens power calculation. J Cataract Refract Surg. 1992;18:125-129.

3. Lee AC, Qazi MA, Pepose J. Biometry and intraocular lens power calculation. Curr Opin Ophthalmol. 2008;19:13-17.

4. Olsen T. The accuracy of ultrasonic determination of axial length in pseudophakic eyes. Acta Ophthalmol. 1989;67:141-144.

5. Olsen T. Theoretical approach to intraocular lens calculation using Guassian optics. J Cataract Refract Surg. 1987;13:141-145.

6. Findl O, Drexler W, Menapace R, et al. High precision biometry of pseudophakic eyes using partial coherence interferometry. J Cataract Refract Surg. 1998;24:1087-1093. 
7. Haigis W. Optical coherence biometry. Dev Ophthalmol. 2002;34: 119-130.

8. Németh J, Fekete O, Psztenlehrer N. Optical and ultrasound measurement of axial length and anterior chamber depth for intraocular lens power calculation. J Cataract Refract Surg. 2003;29:85-88.

9. Packer M, Fine IH, Hoffman RS, et al. Immersion A-scan compared with partial coherence interferometry; outcomes analysis. J Cataract Refract Surg. 2002;28:239-242.

10. Findl O, Kriechbaum K, Sacu S, et al. Influence of operator experience on the performance of ultrasound biometry compared to optical biometry before cataract surgery. J Cataract Refract Surg. 2003;29:1950-1955.

11. Vogel A, Dick HB, Krummenauer F. Reproducibility of optical biometry using partial coherence interferometry: intraobserver and interobserver reliability. J Cataract Refract Surg. 2001;27:1961-1968.

12. Hitzenberger CK, Drexler W, Dolezal C, et al. Measurement of the axial length of cataract eyes by laser Doppler interferometry. Invest Ophthalmol Vis Sci. 1993;34:1886-1893.
13. Kiss B, Findl O, Menapace R, et al. Refractive outcome of cataract surgery using partial coherence interferometry and ultrasound biometry: clinical feasibility study of a commercial prototype II. $J$ Cataract Refract Surg. 2002;28:230-234.

14. Haigis W, Lege B, Miller N, Schneider B. Comparison of immersion ultrasound biometry and partial coherence interferometry for intraocular lens calculation according to Haigis. Graefes Arch Clin Exp Ophthalmol. 2000;238:765-773.

15. Reddy AR, Pande MV, Finn P, El-Gogary H. Comparative estimation of anterior chamber depth by US, Orbscan II, and IOLMaster. $J$ Cataract Refract Surg. 2004;30:1268-1271.

16. Freeman G, Pesudovs K. The impact of cataract severity on measurement acquisition with IOL Master. Acta Ophthalmol Scand. 2005;83:439-442.
Clinical Ophthalmology

\section{Publish your work in this journal}

Clinical Ophthalmology is an international, peer-reviewed journal covering all subspecialties within ophthalmology. Key topics include: Optometry; Visual science; Pharmacology and drug therapy in eye diseases; Basic Sciences; Primary and Secondary eye care; Patient Safety and Quality of Care Improvements. This journal is indexed on

\section{Dovepress}

PubMed Central and CAS, and is the official journal of The Society of Clinical Ophthalmology (SCO). The manuscript management system is completely online and includes a very quick and fair peer-review system, which is all easy to use. Visit http://www.dovepress.com/ testimonials.php to read real quotes from published authors. 